\title{
Risk of appendicitis after endoscopic full-thickness resection of lesions involving the appendiceal orifice: a retrospective analysis $\square$
}

\begin{abstract}
Authors Meining ${ }^{4}$, Karel Caca ${ }^{2}$

Institutions

1 Universitätsklinik Ulm, Klinik für Innere Medizin I, Ulm, Germany

2 Klinikum Ludwigsburg, Klinik für Innere Medizin, Gastroenterologie, Hämato-Onkologie, Pneumologie, Diabetologie und Infektiologie, Ludwigsburg, Germany

3 Kliniken des Landkreises Neumarkt i.d. OPf., Neumarkt, Germany

4 Universitätsklinikum Würzburg, Medizinische Klinik und Poliklinik II, Gastroenterologie, Würzburg, Germany
\end{abstract}

Simone Schmidbaur ${ }^{*}, 1$, Andreas Wannhoff², Benjamin Walter ${ }^{*}, 1$, Benjamin Meier ${ }^{2}$, Claus Schäfer ${ }^{3}$, Alexander

submitted 23.11.2019

accepted after revision 13.7.2020

published online 7.9 .2020

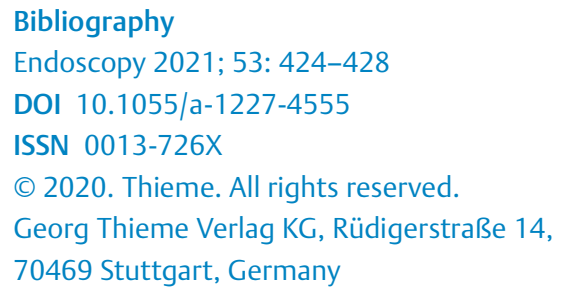

\author{
丹 Supplementary material \\ Supplementary material is available under \\ https://doi.org/10.1055/a-1227-4555
}

\section{ABSTRACT}

Background Conventional endoscopic resection of lesions affecting the appendiceal orifice is difficult. Endoscopic full-thickness resection (EFTR) is a novel technique in interventional endoscopy. As EFTR near the appendiceal orifice is associated with a subtotal appendectomy, it remains unclear whether the risk of developing appendicitis is increased. We conducted a retrospective analysis of lesions involving the appendiceal orifice treated by EFTR.

Methods This was a multicenter retrospective analysis of patients $(n=50)$ treated with EFTR for lesions involving the appendiceal orifice between 2014 and 2019. The objective was to evaluate the occurrence of appendicitis.

Results Acute appendicitis occurred in seven patients (14\%) during follow-up. Conservative treatment was sufficient in four cases, and three patients underwent appendectomy.

Conclusions EFTR of lesions involving the appendiceal orifice may be associated with an imminent risk of developing appendicitis and a consecutive need for appendectomy. Patients should be informed about this specific risk prior to resection. It is unclear why some patients develop appendicitis while the majority remains asymptomatic.

\section{Introduction}

Endoscopic mucosal resection (EMR) and endoscopic submucosal dissection are highly effective techniques for resection of colorectal lesions. However, they have several limitations [1]. In particular, EMR of lesions or laterally spreading lesions involving the appendiceal orifice is technically demanding ( $\mathbf{F i g . 1}$ ) due to difficult access to the appendiceal lumen and a high risk of perforating the thin cecal wall. Therefore, surgical intervention is often necessary [2,3]. To allow definitive diagnosis and

\footnotetext{
* These authors contributed equally to this work.
}

treatment, an endoscopic full-thickness resection (EFTR) device (Ovesco Endoscopy, Tübingen, Germany) had been developed. It enables the endoscopist to perform EFTR with immediate defect closure [4]. The system is suitable for resection in difficult anatomical places with high risk of perforation, such as in para-diverticular or para-appendicular disease [2,5]. In addition, the prompt closure provided by the EFTR device had been described as minimizing the risk of peritoneal irritation during resection by shortening the contact time between bowel lumen and peritoneal cavity [6]. To date, the largest published study dealing with colonic EFTR included 181 patients and reported $\mathrm{R} 0$ resection rates of $77.7 \%$ and $72.4 \%$ for diffi- 
cult-to-resect colorectal lesions and early cancers, respectively [1].

As EFTR affecting the appendiceal orifice is associated with subtotal appendectomy, it remains unclear whether the risk of developing appendicitis is increased (see Fig. $\mathbf{1} \mathbf{s}$ in the onlineonly supplementary material). The aim of this retrospective study was to further evaluate the risks associated with EFTR at this challenging location.

\section{Methods}

All procedures were performed in an inpatient setting with patients under propofol sedation with or without midazolam, and with $\mathrm{CO}_{2}$ inflation. Lesion diameter and extension into the appendix were estimated during an initial endoscopy. After marking lateral lesion margins, the endoscope was equipped with the EFTR device and advanced to the lesion. The colonic EFTR device was mounted onto a standard colonoscope (diameter $11.5-13.2 \mathrm{~mm}$ ). The device consists of a transparent cap with a pre-assembled over-the-scope (OTS) clip device. The tip of the cap harbors a polypectomy snare, which runs along the outside of the scope covered by a sleeve to prevent entrapment of tissue between the snare and scope. The specimen was pulled into the cap by grasping forceps. The OTS clip was deployed by turning the hand wheel on the endoscope handle, and the so created pseudopolyp, with the targeted tissue above the clip, was removed using the preloaded snare. Thereafter, the resected specimen was captured within the cap and withdrawn [7] ( $>$ Fig. 2, V Video 1). For lesions $>2 \mathrm{~cm}$, which are too big to be resected via the EFTR method alone, a hybrid technique consisting of EFTR and EMR (hybrid EMR-EFTR) was used. For the present study, all cases of colonic EFTR performed at three participating endoscopy centers (Ludwigsburg, Neumarkt, Ulm) between 2014 and 2019 were retrospectively reviewed.

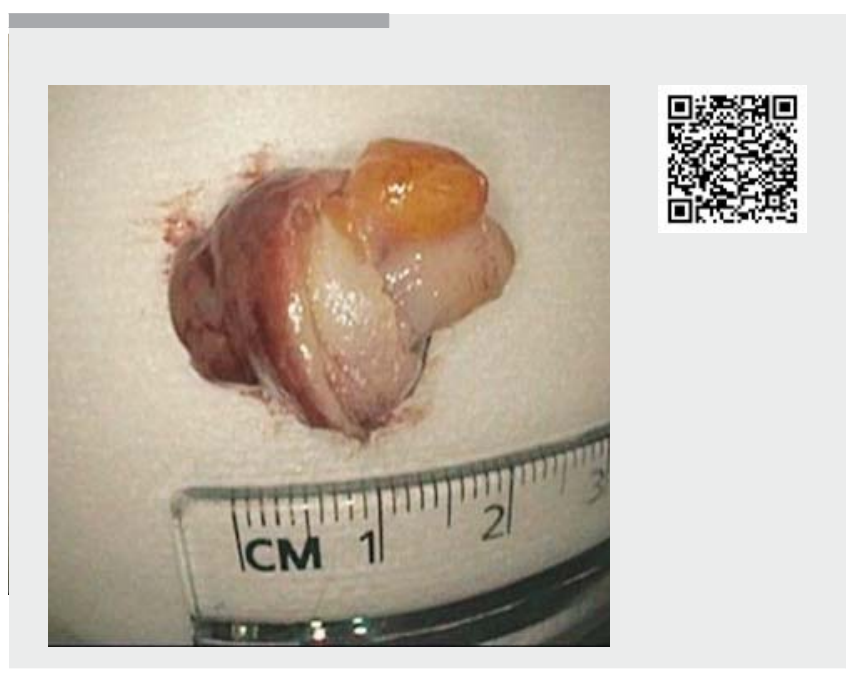

Video 1 Full-thickness resection of a lesion affecting the appendiceal orifice.

Online content viewable at:

https://doi.org/10.1055/a-1227-4555
The rate of development of post-interventional appendicitis leading to possible indication for surgical appendectomy was defined as the primary end point. Post-interventional appendicitis was defined as inflammatory response of the residual appendix with characteristic clinical signs, symptoms, and laboratory results. Abdominal sonography was possible but not mandatory for diagnosis.

Resection state and histopathology of resected specimens were also analyzed. Resection state was defined using the $R$ classification [8]. R0 histology was defined as "no residual tumor," R1 as "microscopically detected residual tumor," and Rx as "evaluation regarding residual tumorous tissue not possible."

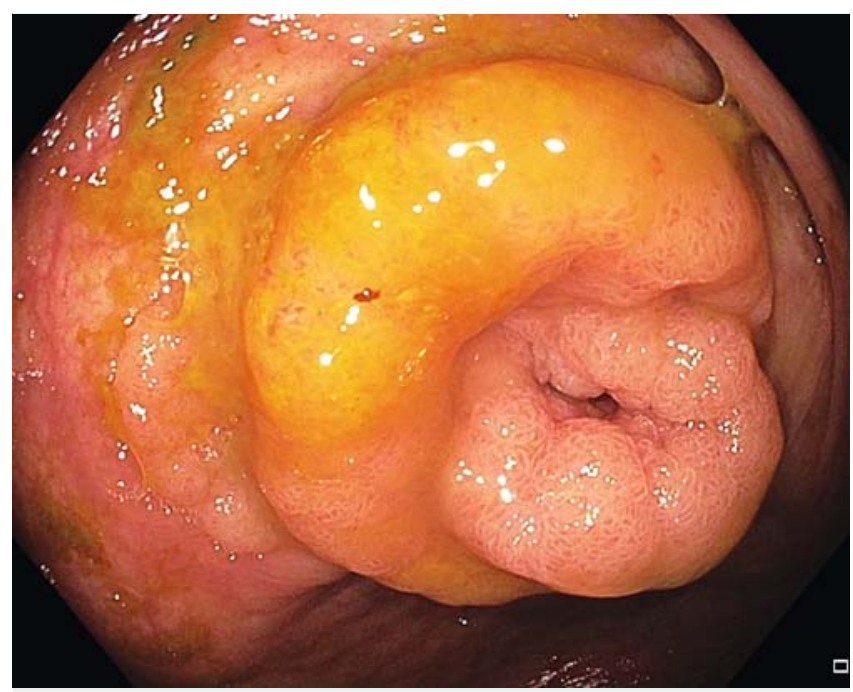

> Fig. 1 Adenoma affecting the appendiceal orifice.

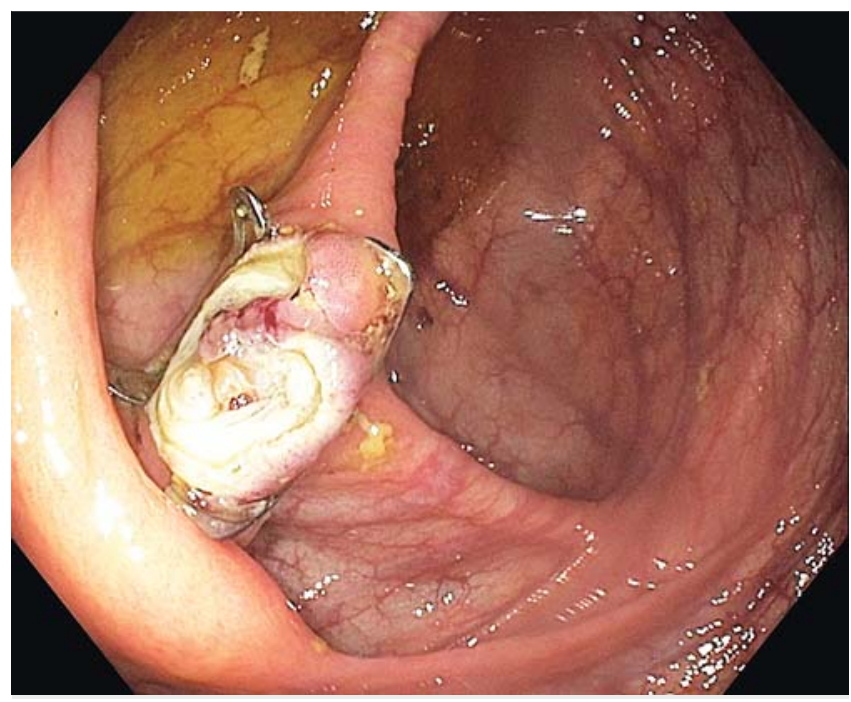

Fig. 2 Area of adenoma near the appendiceal orifice resected by endoscopic full-thickness resection including subtotal appendectomy. 
Descriptive statistical analysis was performed on the data using SPSS Statistics version 25 (IBM Corp., Armonk, New York, USA).

\section{Results}

Resection of lesions at the appendix was performed in 56 patients by expert endoscopists. Six of the 56 patients were excluded from the analysis owing to prior appendectomy. Of the 50 remaining patients, EFTR was technically successful in 48 (96\%) ( Table 1). In two cases, deployment of the OTS clip was not possible due to a highly angulated colonoscope; a standard resection (EMR) was successfully performed in these cases. Follow-up was terminated after a mean of 4 months.

All patients were sedated with propofol ( \pm midazolam) and received antibiotics peri-interventionally for a mean of 4 days. R0 resection was achieved in 32 patients (64\%), Rx in 15 (30 $\%)$, and $R 1$ in 3 (6\%). In 7 of the 15 cases with an Rx result, the lesions were resected using the hybrid EMR-EFTR technique. Histology results are presented in $>$ Table 1 and $>$ Fig. 3. In five cases, histology showed no adenomatous tissue in the resected specimen, although indication for resection was given macroscopically.

Post-interventional appendicitis occurred in seven patients (14\%), four of whom were managed conservatively with intravenous hydration, antibiotics ( $3-5$ days) and analgesics ( $\triangleright$ Table 2). Three patients with appendicitis underwent surgical appendectomy. A bimodal distribution of the onset of appendicitis was observed: four appendicitis cases occurred during the first 10 days of post-interventional monitoring; another three occurred after a latency period of $<1$ month after the procedure. Patients with uncomplicated appendicitis received conservative treatment. Patients with increased risk for perforation underwent primary surgery. Therapeutic decisions were made by visceral surgeons and gastroenterologists.

Three out of the four patients ( $75 \%$ ) with appendicitis during the first 10 days after the procedure were treated by antibiotic administration, and the other patient underwent surgery $(\triangleright \mathrm{Ta}$ ble 2). In contrast, two out of the three patients (67\%) with appendicitis within $<1$ month needed surgical therapy.

In one case of sessile serrated adenoma, post-interventional perforation of the cecum occurred and was treated surgically ( Table 1). No other post-procedural complications (bleeding, severe pain, unexpected hospitalization) were reported.

In conclusion, $92 \%$ of cases with lesions involving the appendiceal orifice were treated using an EFTR technique alone instead of surgery.

\section{Discussion}

EFTR is an emerging technique for the resection of epithelial and subepithelial lesions throughout the gastrointestinal tract. The resection of lesions at the appendix is often considered a controversial issue due to a high risk of perforation or R1/Rx resection. Conventionally, patients are referred for surgical resection. In this retrospective multicenter study on EFTR of lesions involving the appendiceal orifice, 50 cases were analyzed.
- Table 1 Patient and treatment characteristics.

\begin{tabular}{|c|c|}
\hline Patients*, n & 50 \\
\hline \multicolumn{2}{|l|}{ Sex, n (\%) } \\
\hline - Male & $16(32)$ \\
\hline - Female & $34(68)$ \\
\hline Age, mean (range), years & $65.8(46-83)$ \\
\hline Endoscopic accessibility of lesion site, $n$ (\%) & $50(100)$ \\
\hline Technically successful intervention, n (\%) & $48(96)$ \\
\hline Lesion size, mean (SD), mm & $18.3(10.6)$ \\
\hline Prophylactic antibiotics, mean, days & 4 \\
\hline \multicolumn{2}{|l|}{ R status, n (\%) } \\
\hline - RO & $32(64)$ \\
\hline - $\mathrm{Rx}$ & $15(30)$ \\
\hline - R1 & $3(6)$ \\
\hline \multicolumn{2}{|l|}{ Histopathology, n (\%) } \\
\hline - Sessile serrated adenoma & $22(44)$ \\
\hline - Tubular adenoma LGIEN & $13(26)$ \\
\hline - Tubular adenoma HGIEN & $2(4)$ \\
\hline - Tubulovillous adenoma LGIEN & $4(8)$ \\
\hline - Tubulovillous adenoma HGIEN & $3(6)$ \\
\hline - Adenocarcinoma & $1(2)$ \\
\hline - No adenoma & $5(10)$ \\
\hline \multicolumn{2}{|l|}{ Adverse events, n (\%) } \\
\hline - Post-interventional appendicitis & $7(14)$ \\
\hline - Perforation of cecum & $1(2)$ \\
\hline \multicolumn{2}{|c|}{ Treatment of adverse events, $\mathrm{n}$ (\% of adverse events) } \\
\hline - Conservative & $4(50)$ \\
\hline - Surgical & $4(50)$ \\
\hline $\begin{array}{l}\text { SD, standard deviation; LGIEN, low grade intraepi } \\
\text { high grade intraepithelial neoplasia. } \\
\text { * Patients with prior appendectomy were exclude }\end{array}$ & eoplasia; HGIEN, \\
\hline
\end{tabular}

The target lesion could be reached in all cases, although advancing the endoscope equipped with the sturdy EFTR device can be problematic. Nevertheless, a technical resection success rate of $96 \%$ was achieved. However, the R0 resection rate was only $64 \%$, which is lower than that reported by other authors [9]. The comparably high rate of Rx or R1 histology might be explained by the difficulty in assessing the depth of infiltration into the appendiceal lumen. We therefore conclude that visualization of adenoma margins should be mandatory when using this technique.

Rx histology in 7 of the 15 cases might have been due to the use of the hybrid EMR-EFTR technique, as the combination of EFTR and piecemeal EMR of bigger lesions may impede histological assessment of the $R$ status. Precise follow-up examina- 


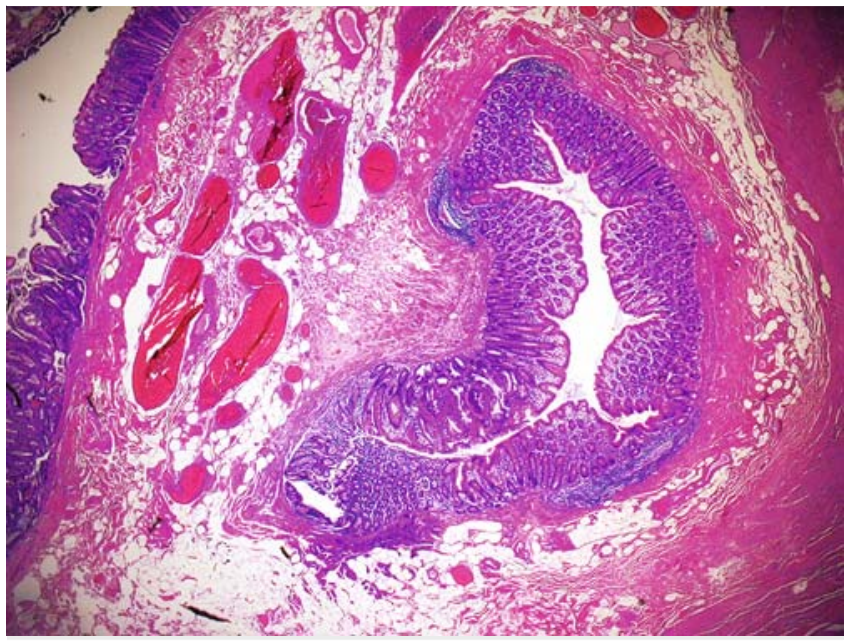

- Fig. 3 Histopathologic specimen showing a sessile serrated adenoma (on the left of the image); the appendix in submucosal tissue after subtotal appendectomy can also be seen (right of center).

tions to evaluate residual and/or recurrent polypoid tissue are therefore mandatory. If residual or recurrent lesions are detected, repeat EFTR, conventional techniques or surgery might be necessary. In previous studies, R0 resection of recurrent or residual lesions with the EFTR system was possible in $77.7 \%$ [1].

Complications were managed surgically in four patients ( 8 $\%)$; therefore, EFTR presented as an alternative to primary surgery in $92 \%$ of cases. However, Rx or R1 procedures may require follow-up surgical therapy. This effect was not monitored in our study and could therefore lead to a bias regarding recurrent lesions, as follow-up was terminated after a mean of 4 months. Extended follow-up is crucial for future evaluation. Furthermore, the retrospective design of the study and lack of a control group impairs study validity.
As tumor size is a major limitation of conventional EFTR, the hybrid EMR-EFTR technique seems to be an effective approach for larger lesions [10]. However, two patients treated with hybrid EMR-EFTR developed post-procedural appendicitis. As the case number is too small to derive significant data, evaluation of the outcome of different techniques seems warranted.

In addition, histology must be reviewed carefully. In patients diagnosed with carcinoma who undergo an Rx procedure, it must be ensured that no residual neoplastic tissue, which may have been moved during clipping with the EFTR device, is located on the serosal side of the colon [11].

Although all patients received prophylactic antibiotic treatment for a mean of 4 days, seven patients developed secondary appendicitis. Four of these patients $(57 \%$ ) were treated conservatively with antibiotics and were discharged after inpatient monitoring.

A bimodal distribution of appendicitis onset was observed. Data may indicate that early onset and detection of appendicitis was associated with milder disease progression resulting in conservative treatment options. No specific patient risk factors for appendicitis were determined in this study population.

Endoscopists as well as visceral surgeons should be aware of the complications of EFTR, and patients should be informed about the risk of appendicitis and potential need for surgery prior to undergoing the procedure.

In summary, EFTR of lesions near or affecting the appendiceal orifice was associated with an acceptable complication rate. The risk of developing acute appendicitis was $14 \%$; however, more than half $(57 \%)$ of these cases could be treated conservatively owing to prompt detection. Further studies to determine risk factors for development of post-procedural appendicitis are mandatory.

- Table 2 Characteristics of all seven cases with appendicitis.

\begin{tabular}{|c|c|c|c|c|c|c|c|}
\hline & 1 & 2 & 3 & 4 & 5 & 6 & 7 \\
\hline Age, years & 75 & 58 & 51 & 77 & 72 & 71 & 52 \\
\hline Lesion diameter, mm & 30 & 7 & 13 & 10 & 25 & 8 & 10 \\
\hline Resection method used & $\mathrm{EFTR}+\mathrm{EMR}$ & EFTR & EFTR & EFTR & $\mathrm{EFTR}+\mathrm{EMR}$ & EFTR & EFTR \\
\hline Lesion pathology & SSA & SSA & SSA & - & TVA, HG & - & TVA, LG \\
\hline R status & $\mathrm{Rx}$ & R0 & Ro & - & $\mathrm{Rx}$ & - & R0 \\
\hline Time of onset & With latency & With latency & Early & Early & Early & With latency & Early \\
\hline Management & Conservative & Surgery & Conservative & Conservative & Conservative & Surgery & Surgery \\
\hline
\end{tabular}

EFTR, endoscopic full-thickness resection; EMR, endoscopic mucosal resection; SSA, sessile serrated adenoma; -, no adenoma; TVA, tubulovillous adenoma; HG, high grade; LG, low grade. 


\section{Competing interests}

Professor Meining and Professor Caca are consultants for Ovesco. All other authors declare that they have no conflicts of interest.

\section{References}

[1] Schmidt A, Beyna T, Schumacher B et al. Colonoscopic full-thickness resection using an over-the-scope device: a prospective multicentre study in various indications. Gut 2018; 67: 1280-1289

[2] Tate D], Desomer L, Awadie $\mathrm{H}$ et al. EMR of laterally spreading lesions around or involving the appendiceal orifice: technique, risk factors for failure, and outcomes of a tertiary referral cohort (with video). Gastrointest Endosc 2018; 87: 1279-1288.e2

[3] Jacob H, Toyonaga T, Ohara Y et al. Endoscopic submucosal dissection of cecal lesions in proximity to the appendiceal orifice. Endoscopy 2016; 48: 829-836

[4] Schmidt A, Bauerfeind P, Gubler C et al. Endoscopic full-thickness resection in the colorectum with a novel over-the-scope device: first experience. Endoscopy 2015; 47: 719-725
[5] Al-Bawardy B, Rajan E, Wong Kee Song LM. Over-the-scope clip-assisted endoscopic full-thickness resection of epithelial and subepithelial GI lesions. Gastrointest Endosc 2017; 85: 1087-1092

[6] Vitali F, Naegel A, Siebler J et al. Endoscopic full-thickness resection with an over-the-scope clip device (FTRD) in the colorectum: results from a university tertiary referral center. Endosc Int Open 2018; 6: E98-E103

[7] Aepli P, Criblez D, Baumeler S et al. Endoscopic full thickness resection (EFTR) of colorectal neoplasms with the Full Thickness Resection Device (FTRD): clinical experience from two tertiary referral centers in Switzerland. United European Gastroenterol J 2018; 6: 463-470

[8] Wittekind C, Compton C, Quirke P et al. A uniform residual tumor (R) classification. Cancer 2009; 115: 3483-3488

[9] Bronzwaer M, Bastiaansen B, Koens L et al. Endoscopic full-thickness resection of polyps involving the appendiceal orifice: a prospective observational case study. Endosc Int Open 2018; 06: E1112-E1119

[10] Meier B, Caca K, Schmidt A. Hybrid endoscopic mucosal resection and full-thickness resection: a new approach for resection of large nonlifting colorectal adenomas (with video). Surg Endosc 2017; 31: 4268-4274

[11] Dumoulin FL, Gorris DG, Berger $S$ et al. Full-thickness resection with an over-the-scope device: possible translocation of adenoma tissue in a case of an incomplete resection at the appendix. Endosc Int open 2018; 6: E622-E624 